\title{
Advanced Nuclear Energy Sources
}

H uman beings have been seeking a safe way out of their energy predicament. when fossil fuels as the traditional mainstream source of energy are burning out. meanwhile polluting and warming up the whole planet. While emerging renewable energies are still struggling to overcome their own setbacks, nuclear energy. which gives off no greenhouse gases and features great efficiency. stands out as a sustainable, clean and econonically practical replacement. Inevitably, however: it has to face the lurking fears for possible accidents that leads to radioactive nuclear leaks.

Facing with the challenge, researchers at the Chinese Academy of Sciences (CAS) endeavor to solve key problems in the way to safe usage of nuclear energy for social development. Over the past four decades. they have made significant breakthroughs in the field of controlled nuclear fusion. and also found innovative solutions for safe disposal of nuclear wastes and nuclear fission energy.

\section{EAST - A Little Manmade Sun}

Nuclear fusion. the same kind of chain reaction occurring on the Sun that produces the energy for the life on the Earth, is an attractive solution for future energy supplies due to its massive energy output compared with the negligible fuel stock needed. But exactly because of the huge amount of energy it generates within an extremely short moment, the fusion could easily lead to tremendous explosions, and the high temperature would melt down any possible material on the Earth. Therefore, taming the "Sun" is almost a mission impossible.

Science exploration targets not those easy. but hard.

Targeting controlled utilization of nuclear fusion. the CAS Hefei Institutes of Physical Science (CASHIPS) independently designed. constructed. and operated the world's first Experimental Advanced Superconducting Tokamak (EAST, commonly known as the "Little Sun"). First initiated in July 1998, the construction started in October 2000. and was completed in 2006. The device, the first to employ superconducting toroidal and poloidal magnets in the world, aims to obtain plasma pulses sustaining for up to 1.000 seconds. Now it has

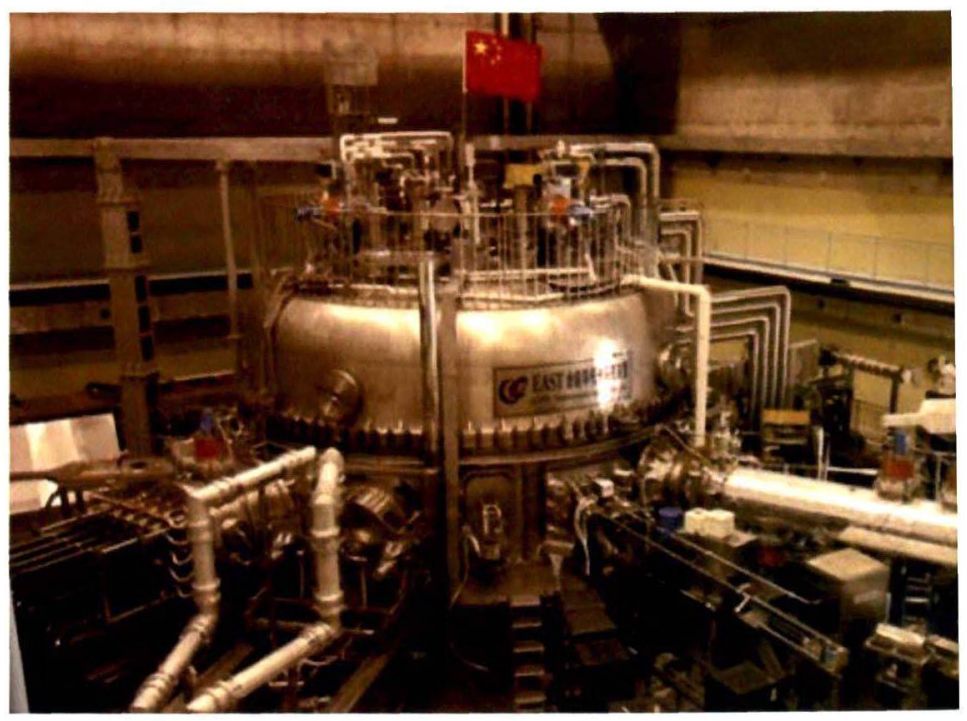

China's Experimental Advanced Superconducting Tokamak (EAST), a nuclear fusion experimental device in Hefei, China. (Credit: CASHIPS)

become an important experimental platform for physies and engineering research in nuclear fusion.

The tokamak reactor consists of a metal torus 
or doughnut that is exhausted to a hard vacuum and then injected with hydrogen atoms. These atoms are then heated by a number of different methods to create a plasma that is then compressed using a series of powerful superconducting magnets. Eventually. the plasma becomes so hot and so condense that the conditions inside the reactor could mimic those found inside the Sun, causing the hydrogen atoms to fuse into helium, releasing tremendous amounts of energy.

The equipment achieved its first plasma on September 26 the same year it was completed. By Jan 2007, the reactor had successfully created a plasma

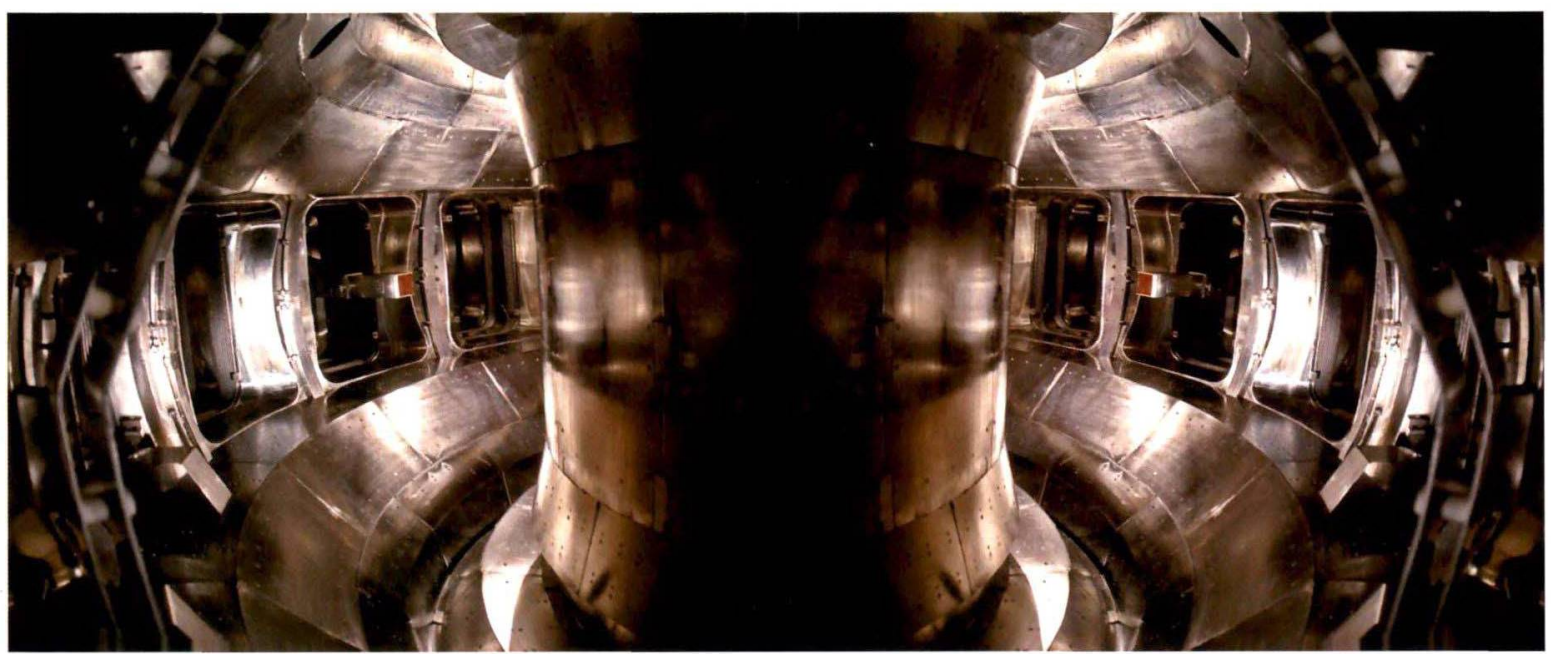

A view of the EAST interior, into which the hydrogen atoms are injected, then heated and compressed to cause fusion, releasing extreme amounts of energy. (Credit: CASHIPS)

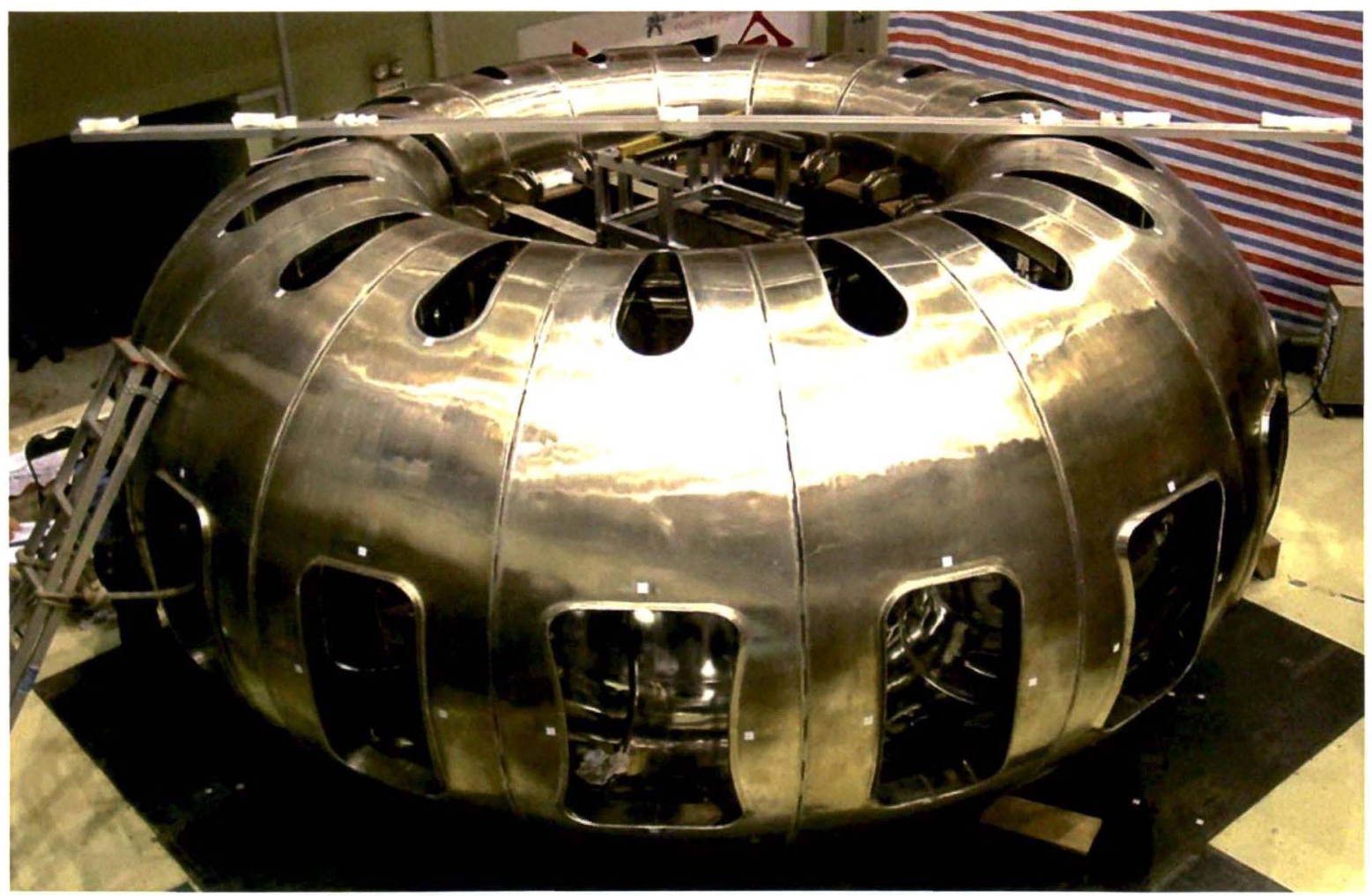

The vacuum vessel ring of EAST as seen in 2004, being installed. (Credit: CASHIPS) 
lasting for nearly five seconds, generating an electrical current of $500 \mathrm{kA}$. In February 2016, a plasma pulse was achieved and maintained for a record 102 seconds at approximately 50 million Kelvin. The plasma current measured $400 \mathrm{kA}$, mounted to a density of about $2.4 \times 10^{18} / \mathrm{m}^{3}$ with slowly increasing temperature.

On July 3. 2017, EAST obtained a fully noninductive current-driven (steady-state) highconfinement plasma for over 100 seconds, setting a new world record. These results have provided experimental support for the long-pulse high-confinement operation of the International Thermonuclear Fusion Experimental Reactor (ITER), and laid a foundation for the design of China's next-generation fusion device, the China Fusion Engineering Experimental Reactor (CFETR).

In 2018, EAST achieved an electron temperature of over 100 million degrees centigrade in its core plasma. This is about seven times hotter than the interior of the Sun, which is about 15 million degrees centigrade. This achievement represents a significant step by China towards tokamak-based fusion energy generation.

This project won the 2007 Outstanding Scientific and Technological Achievement Award of CAS, and a First Prize from the National Awards for Science and Technology Progress, respectively in 2008 and 2013.

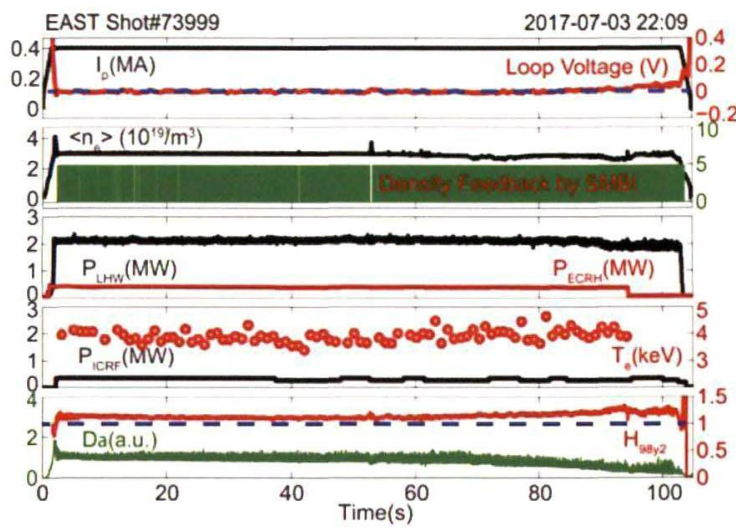

Demonstration of a high-confinement plasma over 100 seconds (Credit: CASHIPS)
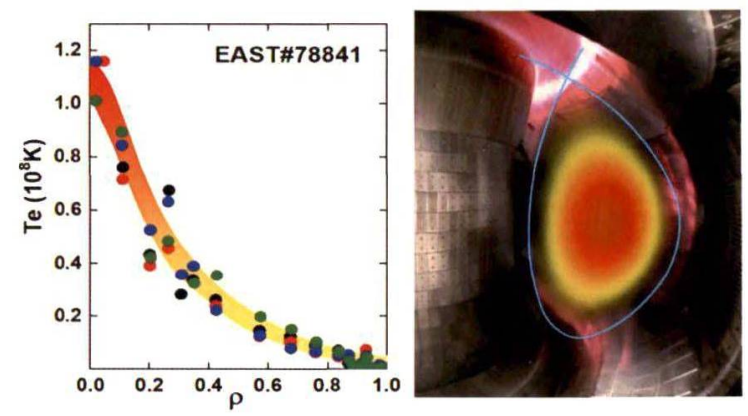

The plasma electron temperature over 100 million degrees centigrade achieved in 2018 on EAST. (Credit: CASHIPS)

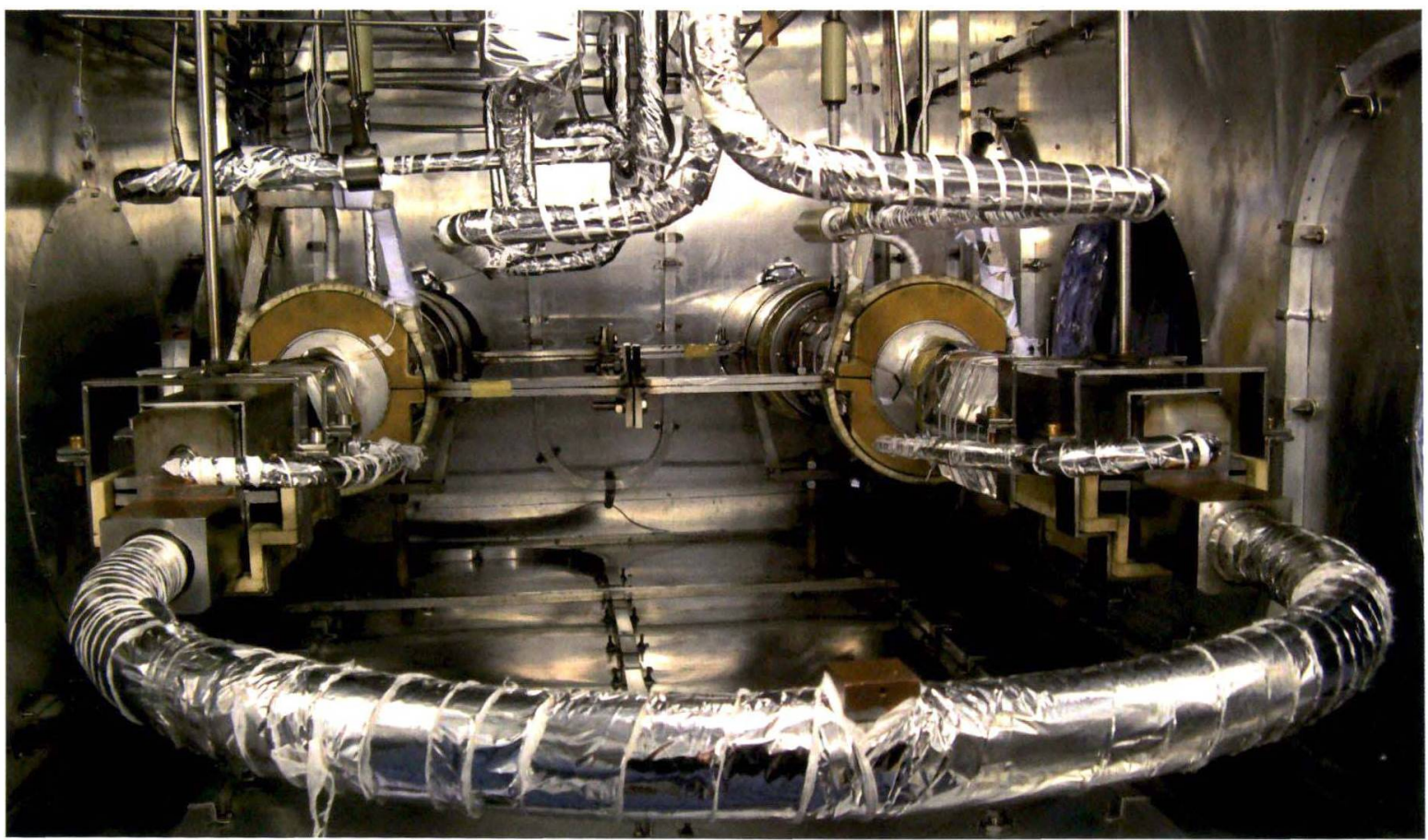

A pair of current leads, made of high-critical-temperature superconducting materials, is being tested in a cryogenic Dewar box. (Credit: CASHIPS) 


\section{ADS: Package Solution for Used Fuel Processing and Energy Generation}

In terms of nuclear fission energy, a headache has been the residual radioactivity in used fuels from reactors. The sustained radioactivity poses a great threat to human health, and their neutralization proves to be highly challenging.

Used fuels. after "burning" in the "furnace" for a period of time, contain a lot of radioactive wastes, particularly minor actinides (MA) and long-lived fission products (LLFP). Some LLFP have half-lives as long as tens of thousands of years, and their complex composition makes the disposal a nightmare. Burying them in the depth of earth will not go on for long: therefore, it has been an imperative issue to develop new technologies to neutralize them.

To address this challenge. in 2011. the CAS Institute of Modern Physics (IMP) began their R\&D to tackle involved key problems, seeking to build an accelerator-driven sub-critical system (ADS). The basic idea of ADS is to design and realize a smart pathway for nuclear reactions that can reduce and neutralize the radioactivity inherent to the used fuels. and meanwhile produce sustained energy by reusing the "used fuels". A rough pathway is to first bombard some heavy nuclei like lead with starter ions using a proton beam from an accelerator. to produce a large number of neutrons. These neutrons are then channeled to a sub-critical reactor, where the nuclear wastes will absorb the neutrons and transmute into low-radioactive substances. In this way, the used fuels will be "burnt out", exhausting their potential as much as possible.

Rosy as it looks. this idea remains a beautiful concept so far, pending for practical solution. despite decades of efforts by different teams across the world.

After years work, in 2016, the IMP team first proposed a solution for ADS and based on it built a prototype system. The IMP solution can increase the utilization rate of uranium resources from less than $1 \%$ to over $95 \%$, greatly exploiting the residual value of used fuels. More encouragingly, the nuclear waste output accounts for only $4 \%$ of the input used fuels: and the half-lives of the processed used fuels could be reduced from tens of thousands of years to about 500 vears.

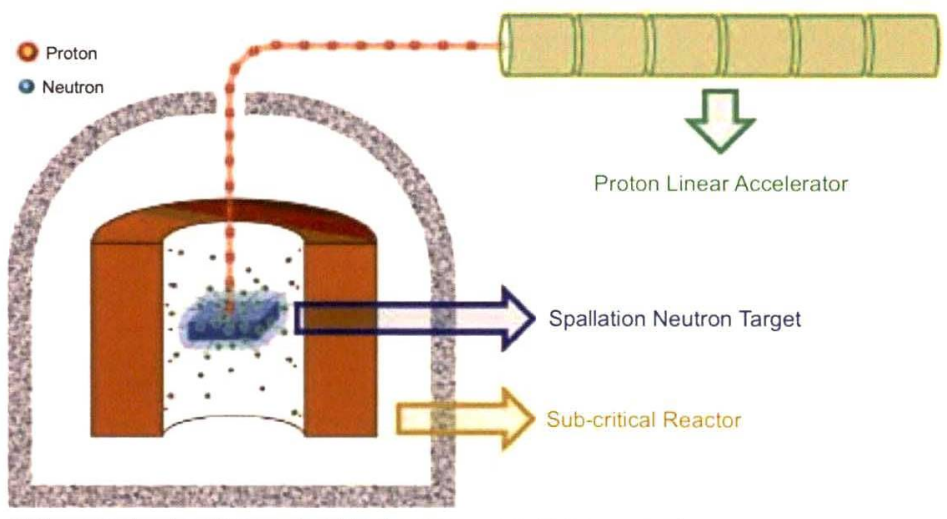

ADS - accelerator-driven sub-critical system - could be a smatt way for safe disposal of nuclear wastes.

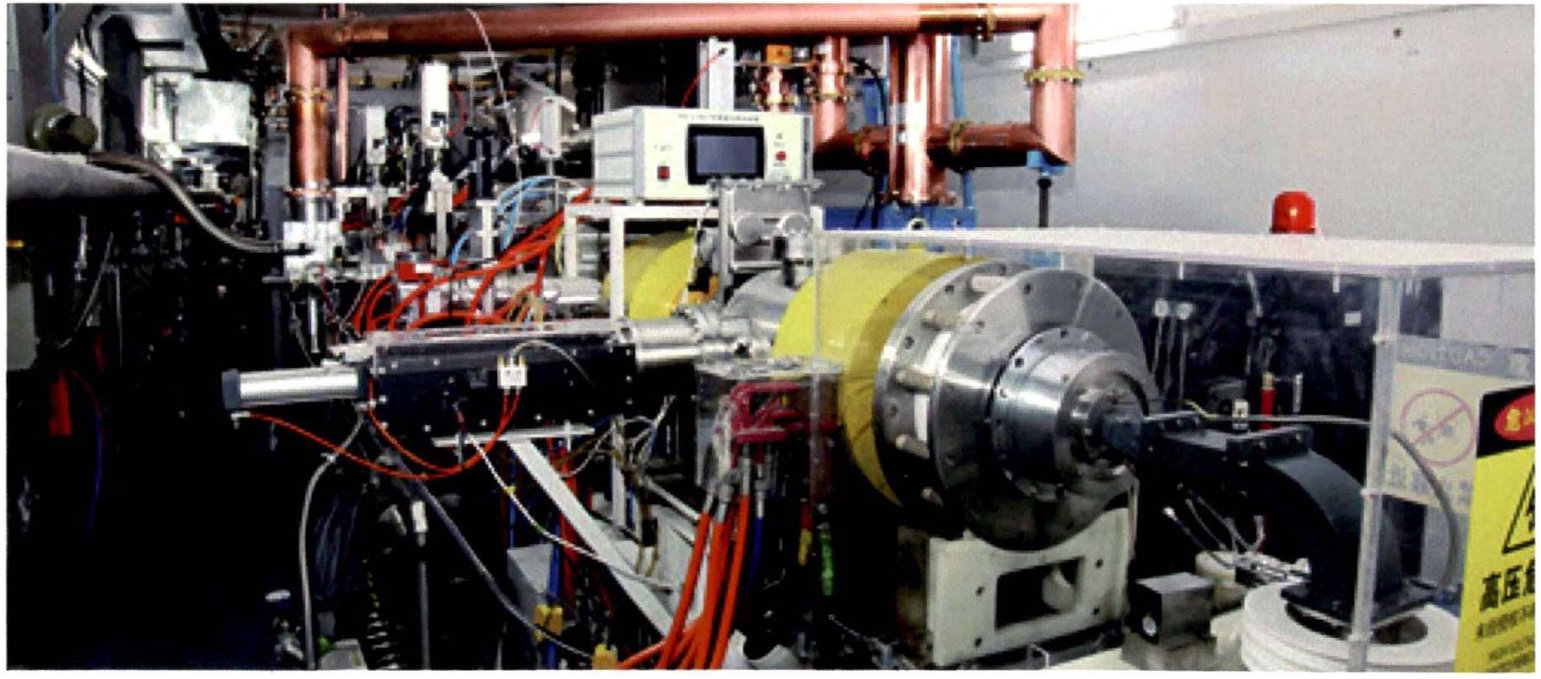

The "starting gun" of ADS: the superconductive proton linear accelerator that feeds the system with ion sources (Credit: IMP) 


\section{TMSR: New Strategy for Safe Nuclear Fission Energy}

Thorium molten salt reactor (TMSR), based on a thorium/uranium fuel cycle. comes back to light from negligence as an essentially infallible energy strategy in recent vears. Unlike the current mainstream reactors using uranium/plutonium fuels, according to experts. in a TMSR no spontaneous chain reaction would be sparked. nor will a meltown happen in the case of cooling system failure. This helps quiet the fears for possible nuclear leaks as a consequence from a potential natural catastrophe - like the Fukushima Daiichi nuclear crisis broke out in March 2011 in the wake of a massive earthquake of magnitude 9.0 and a subsequent tsunami.

In the case of TMSR, fuels are melted in hot fluorides, which at the same time serve as a coolant recvcling in the inner and outer circles within the reactor. The kind of overheating in Fukushima will not occur here because once the temperature at the core goes bevond a threshold. a "freeze plug" at the bottom of the reactor core will melt down itself to drain the molten salt off an emergency dump tank connected to the core. and thus stop the reaction.

On top of the safety. thorium-based fuels offer better productivity for energy generation: the higher abundance and even distribution of thorium in the Earth's crust also add to the advantages. Moreover. TMSR discharges no plutonium. a well-known material for nuclear weapons: and either of its main products. the uranium 2:3:3 and the uranium $2: 32$ is suitable for nuclear weapon manufacture use. Therefore. its application will not result in nuclear proliferation.

All this said. before we can use TMSR for our energy supplier, a series of scientific issues have to be solved, particularly those concerning quick preparation of fuels, and safe disposal of the radioactive wastes.

To break through the bottleneck. the Shanghai Institute of Applied Physics (SINAP). CAS took the challenge and conducted related RA.D) to tackle key technological problems in design. construction and operation of a TMSR system. The progress they made so far has paved the way for building an experimental reactor:

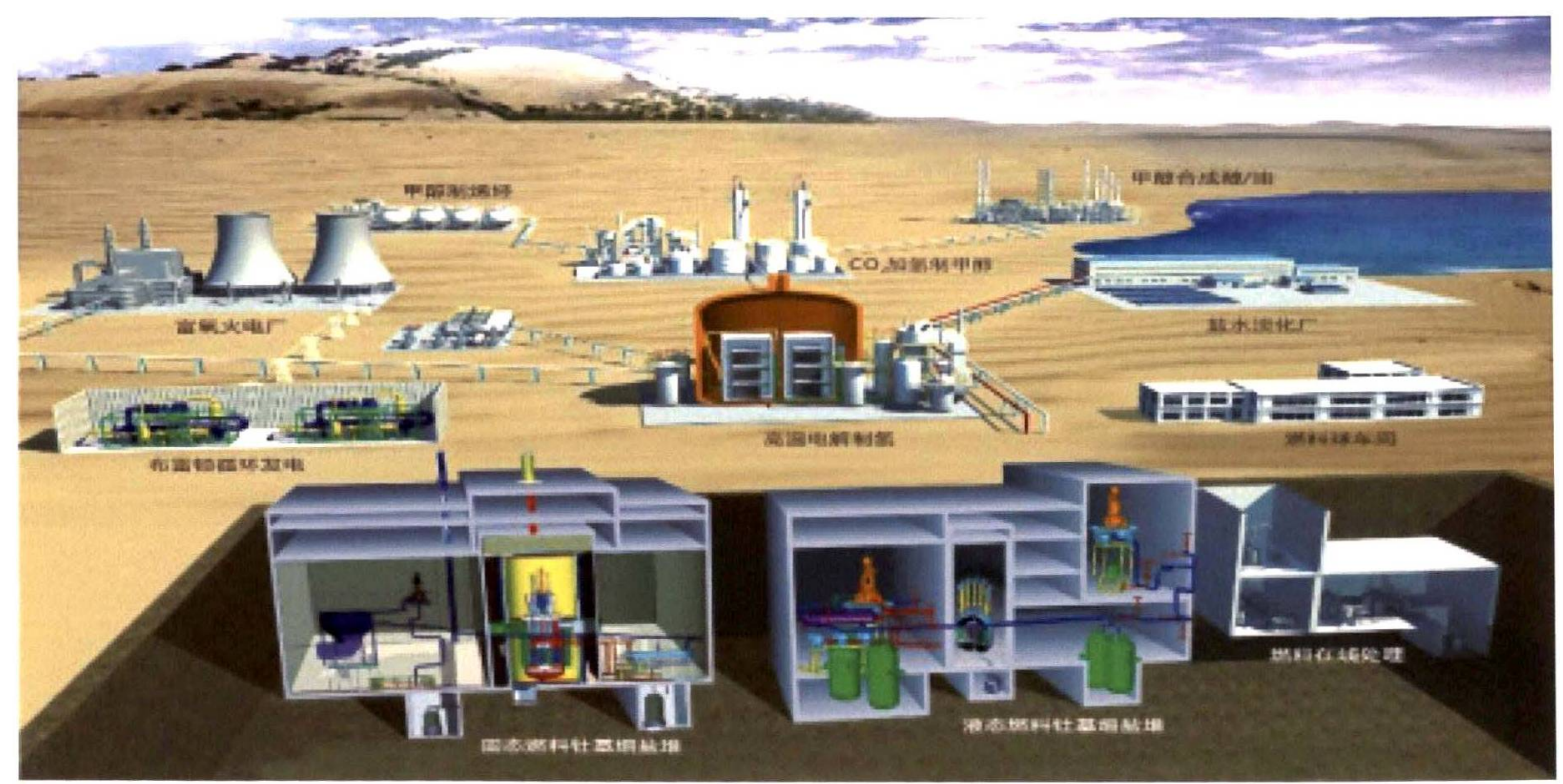

A schematic design for a TMSR base (Credit: SINAP) 